\title{
Aplicación de la metodología ApS en alumnos del Grado de Logopedia: intervención con personas de la tercera edad.
}

Teresa Perez Gil ${ }^{\mathrm{a}}$, Claudia Lahiguera Lujan ${ }^{\mathrm{b}}, \mathbf{M}^{\mathrm{a}} \mathrm{Jose}$ Bautista Pérez${ }^{\mathrm{b}}$, Soraya Triguero Tarazona $^{\mathrm{b}}$, Sheila Baguena Mancebo ${ }^{\mathrm{b}}$, y Dolores Ortiz-Masià ${ }^{\mathrm{c}}$

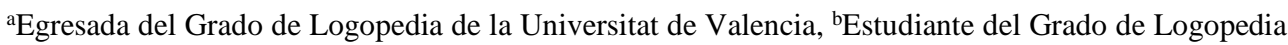
de la Universitat de Valencia y 'Profesora Ayudante Doctora, Departamento de Medicina. Facultad de Medicina y Odontología. Universitat de Valencia (m.dolores.ortiz@uv.es).

\begin{abstract}
Service-learning (SL) is a methodology that contributes to the formation of values and the citizenship of university students, who can acquire a relevant role in the construction of a more dignified, inclusive, cohesive and equitable citizenship. The objective of the project is to relate and integrate the curricular objectives of the subject "Clinical Neurology applied to Speech Therapy" and the objectives of the community's service. The proposed activity consisted of Speech Therapy second year students implementing the basic clinical propaedeutic (anamnesis, neurological and cognitive exploration) in elderly people, all framed in a service-learning activity. Some Speech therapy students and the professor responsible of the subject guided the interventions. The interventions were previously reviewed and practiced in the practical seminars. Once the intervention was done, the students made reports with their conclusions, which were evaluated by the teacher and the collaborating students.
\end{abstract}

Keywords: speech therapy, service-learning, senior citizens, clinical propaedeutic, evaluation.

\section{Resumen}

El aprendizaje-servicio (ApS) es una metodología que contribuye a la formación en valores y para la ciudadanía de los estudiantes universitarios, los cuales pueden adquirir un papel relevante en la construcción de una ciudadanía más digna, inclusiva, cohesionada y equitativa. El objetivo del proyecto es relacionar e integrar los objetivos curriculares de la asignatura "Neurología Clínica aplicada a la Logopedia" y los objetivos de servicio a la comunidad. La actividad propuesta consistió en que alumnos de $2^{\circ}$ de Logopedia implementen la propedéutica clínica básica (anamnesis, exploración neurológica y cognitiva) en personas de la tercera edad, todo ello encuadrado en una actividad de aprendizaje-servicio. Cada una de las intervenciones fueron guiadas por alumnos colaboradores de $3^{\circ}$ de Logopedia $y$ por el profesor responsable de la asignatura. Las intervenciones fueron previamente revisadas, y practicadas en los seminarios prácticos. Una vez realizada la intervención, los alumnos realizaron informes con sus conclusiones, que fueron evaluadas por el profesor y los alumnos colaboradores. 
Palabras clave: logopedia, aprendizaje-servicio, tercera edad, propedéutica clínica, evaluación.

\section{Introducción}

La formación ética y en valores, en los estudiantes universitarios, es necesaria para construir una ciudadanía interactiva e inclusiva. Por otro lado, el aprendizaje-servicio (ApS), conocido internacionalmente como Service learning (SL), es una metodología que contribuye a la formación en valores y para la ciudadanía de los estudiantes universitarios, los cuales pueden adquirir un papel relevante en la construcción de una ciudadanía más digna, inclusiva, cohesionada y equitativa (Martínez, 2010). Los proyectos de ApS permiten a los participantes aplicar los conocimientos adquiridos en las aulas al servicio de necesidades concretas de la comunidad (Puig, Batlle, Bosch y Palos, 2007).

Dentro de los programas de ApS se pueden distinguir cuatro tipos de servicios en los que concretan el aprendizaje: servicio directo, servicio indirecto, investigación y advocacy (Casado de la Gala, 2015).

La formación de Grado en Logopedia está regulada por la ORDEN CIN/726/2009, de 18 de marzo, donde se establecen los objetivos y competencias que los egresados deben tener para el correcto desarrollo de la profesión. Dentro de los objetivos que cita dicha ORDEN se encuentra el objetivo "Explorar, evaluar, diagnosticar y emitir pronóstico de evolución de los trastornos de la comunicación y el lenguaje desde una perspectiva multidisciplinar, fundada en la capacidad de interpretación de la historia clínica para lo que se aplicarán los principios basados en la mejor información posible y en condiciones de seguridad clínica". El citado objetivo se implementa de forma concreta durante el desarrollo de la asignatura “Neurología Clínica Aplicada a la Logopedia”. Para alcanzar el objetivo se deben adquirir competencias muy relacionadas con la evaluación y diagnóstica (propedéutica clínica básica) tales como:

- Conocer los fundamentos del proceso de evaluación y diagnóstico.

- Conocer y aplicar los modelos, técnicas e instrumentos de evaluación.

- Realizar la evaluación de las alteraciones del lenguaje en los trastornos específicos del desarrollo del lenguaje como las alteraciones del lenguaje en el envejecimiento y los trastornos degenerativos.

- Redactar un informe de evaluación logopédica.

- Realizar una evaluación tras la intervención.

La asignatura, dentro del plan de estudios, se encuentra en segundo. En ese mismo curso se inician las prácticas académicas externas que permiten aplicar y complementar los conocimientos adquiridos en la formación académica, favoreciendo la adquisición de competencias que preparan para el ejercicio de actividades profesionales (reguladas por el Real Decreto 592/2014, de 11 de julio).

En el Departamento de Medicina, de la Universidad de Valencia, en el curso 2017-2018 se ha implantado una metodología docente adicional a las prácticas externas. Con el fin de potenciar la adquisición de las competencias propias de la asignatura se pretende incorporar

(cc) EY-NC-ND 2018, Universitat Politècnica de València

Congreso IN-RED (2018) 
actuaciones de servicio voluntario a la comunidad, en el contexto universitario. En la planificación del proyecto de ApS se planteó la intervención de personas de la tercera edad ya que suele ser un colectivo susceptible a alteraciones del lenguaje por el envejecimiento y/o por los trastornos degenerativos.

El proyecto ApS diseñado se encuadra dentro de la categoría de servicio directo donde los objetivos curriculares y los objetivos de servicio se encuentran relacionados e integrados.

\section{Objetivos}

El objetivo general es ofrecer un servicio a la comunidad, y fomentar las enseñanzas que pueden obtener los alumnos de Logopedia a la vez que prestan el servicio. De forma concreta los objetivos que se desean alcanzar con la implantación del proyecto ApS entre los alumnos del Grado de Logopedia son:

- En relación a los estudiantes: formar logopedas competentes para trabajar en residencias de ancianos, desarrollando actitudes favorables a la diversidad, adquisición de destrezas y habilidades técnicas y comunicativas, así como tener una visión global de la práctica profesional del logopeda.

- $\quad$ En relación a las personas de la tercera edad: mejorar la calidad de vida de las personas de la tercera edad con necesidades de acompañamiento, de planificación socio-sanitaria relacionada con alteraciones del lenguaje y en la atención a la dependencia.

- En relación a estudiantes y personas de la tercera edad: potenciar el intercambio de conocimientos intergeneracional.

- En relación a los estudiantes colaboradores de tercer curso y recién egresados: se pretende que adquieran las mismas competencias que los estudiantes de $2^{\circ}$ curso, pero adicionando otras competencias como son el desarrollo del liderazgo, toma de decisiones, y la adquisición de competencias de enseñanza-aprendizaje.

- $\quad$ En relación al profesor de la UV: realizar un análisis de la implantación de un proyecto de ApS en alumnos de segundo de Logopedia y comparar los resultados con alumnado que no recibió esta metodología docente.

\section{Desarrollo de la innovación}

El presente proyecto se centra en una propuesta de intervención basada en la metodología educativa del Aprendizaje-Servicio para la asignatura Neurología Clínica aplicada a la Logopedia impartida en el $2^{\circ}$ curso del Grado de Logopedia. El proyecto se encuentra dentro del programa B, Renovación de metodologías docentes en el marco de las ayudas para el desarrollo de proyectos de innovación educativa y mejora de la calidad docente de la Universitat de Valencia. Dentro del programa B, el proyecto se encuentra en la categoría RMD-ESTIC, donde el equipo de trabajo está formado por un profesor docente investigador de la UV (PDI) y cuatro estudiantes del Grado de Logopedia y un estudiante de Logopedia recién egresado. El objetivo de este tipo de ayudas se centra en el trabajo cooperativo del proceso enseñanza-aprendizaje, con acciones de soporte y orientación del estudiante en su proceso de aprendizaje. 
En la ejecución del proyecto, se aspira a implantar la metodología de ApS como complemento de los créditos prácticos de la asignatura "Neurología Clínica aplicada a la Logopedia” mediante la aplicación práctica de los conocimientos adquiridos en las clases teóricas en personas de la tercera edad (con la colaboración de la Cruz Roja). El proyecto se implementó en sustitución a la sesión de 3 horas de prácticas que se desarrollan de forma ordinaria en las instalaciones de la Facultad de Psicología. Debido al gran volumen de alumnado (unos 40 alumnos por sesión práctica), el proyecto fue una opción voluntaria para los alumnos que deseaban participar. El proyecto se desarrolló para 15 estudiantes. Debido a que el número de solicitudes fue superior al número de plazas ofertadas (45 solicitudes), se procedió al sorteo de las solicitudes. Los alumnos que no deseaban participar o no salieron en el sorteo desarrollaron la práctica de forma habitual, aplicando la propedéutica clínica básica a sus compañeros de clase.

Como se ha mencionado en los objetivos, los estudiantes colaboradores, junto con el profesor responsable, ayudaron en la implementación de los objetivos del proyecto de ApS mediante la realización de videos demostrativos con ayuda de los Técnicos de Innovación Educativa del Servicio de Formación Permanente e Innovación Educativa (SFPIE), fichas de exploración , seminarios sobre propedéutica clínica básica, desplazamiento del alumnado a la sede de la Cruz Roja e implantación del proyecto en las personas de la tercera edad en la sede.

\section{Actividades vinculadas a los objetivos}

1. Los alumnos colaboradores realizaron, junto con el profesor responsable, simulacros didácticos de las intervenciones que debían realizar los estudiantes con las personas de la tercera edad. Los simulacros serán grabados y se utilizarán durante los seminarios prácticos. 2. Los alumnos (por parejas) prepararon y practicaron con sus compañeros -antes de la intervención- los diferentes apartados de la anamnesis y la exploración neurológica, prestando atención a las posibles dificultades y las características de las personas mayores. Durante el seminario práctico resolvieron todas sus dudas e inquietudes.

3. Los 15 alumnos se desplazaron, junto con los alumnos colaboradores y el profesor responsable, a la sede de la Cruz Roja el 22 de febrero donde pusieron en práctica la propedéutica clínica básica con las fichas diseñadas por el profesor y los alumnos colaboradores (ver Figura 1 y 2). El desplazamiento fue en transporte público.

4. Al finalizar la actividad los alumnos realizaron un informe de la actividad en todas sus fases, con el fin de planificar una posible intervención socio-sanitaria.

5. Los alumnos colaboradores participaron activamente en la evaluación del proyecto y de los estudiantes mediante la implementación de fichas de seguimiento y participando en las sesiones de reflexión con las personas mayores. Con el fin de evaluar la mejora docente con respecto a los compañeros que no participaron en el proyecto ApS, los alumnos colaboradores pasaron la misma ficha de seguimiento y participación a 15 alumnos que no asistieron a la sede de la Cruz Roja.

(cc) EY-NC-ND 2018, Universitat Politècnica de València

Congreso IN-RED (2018) 


\section{Análisis estadístico de los datos}

Los datos obtenidos de las diferentes fichas y encuestas se expresaron como media \pm SEM y se compararon mediante la prueba t de Student desapareada. Se consideró que un valor $\mathrm{p}$ $<0.05$ era estadísticamente significativo.

\section{MINI MNETAL STATE EXMINATION (MMSE)}

Folstein et al. (1975), Lobo et al. (1979)/

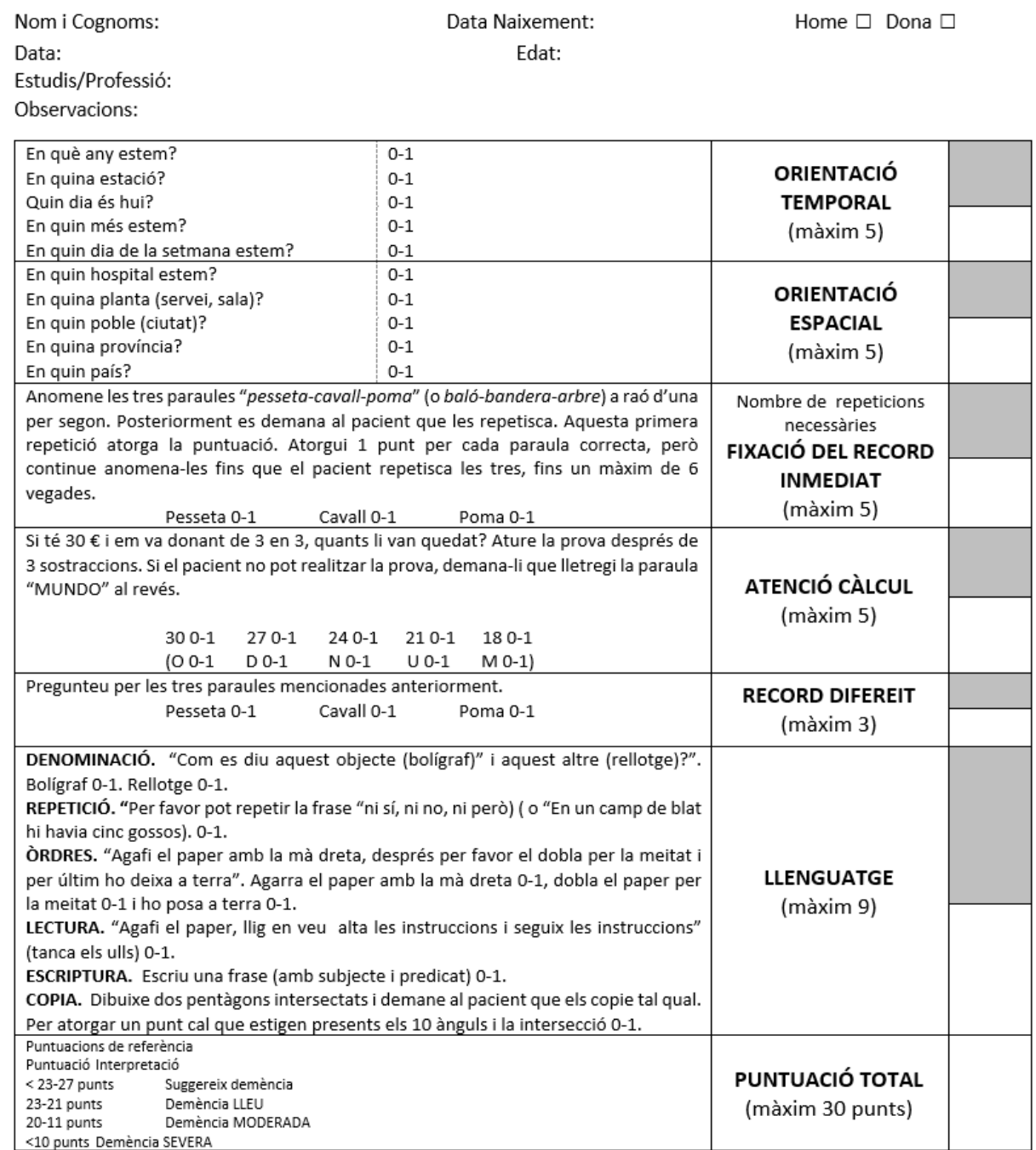

Fig 2. Ficha diseñada para el estudio del estado mental utilizada en la intervención. 
Aplicación de la metodología ApS en alumnos del Grado de Logopedia: intervención con personas de la tercera edad.

\section{EXPLORACIÓ DELS PARELLS CRANIALS DE LA PARLA}

\begin{tabular}{|c|c|c|}
\hline $\begin{array}{l}\text { Nom i Cognoms: } \\
\text { Data: } \\
\text { Estudis/Professió: } \\
\text { Observacions: }\end{array}$ & $\begin{array}{c}\text { Data Naixement: } \\
\text { Edat: }\end{array}$ & Home $\square$ Dona $\square$ \\
\hline \multirow{2}{*}{$\begin{array}{l}\text { PPCC V } \\
\text { TRIGÈMIN }\end{array}$} & $\begin{array}{l}\text { Amb un cotó avalua la sensació al toc suau del front, les galtes i la } \\
\text { mandibula. }\end{array}$ & $\begin{array}{l}\square \text { Nervi oftàlmic (front-ulls-nas) } \\
\square \text { Nervi Maxil-lar (galtes) } \\
\square \text { Nervi Mandibular (mandibula) }\end{array}$ \\
\hline & Demani al pacient que tanqui i obri la mandíbula. & $\begin{array}{l}\square \text { Debilitat } \\
\square \text { Desviació de la mandibula } \\
\square \text { Atròfia }\end{array}$ \\
\hline \multirow{3}{*}{$\begin{array}{l}\text { PPCC VII } \\
\text { FACIAL }\end{array}$} & $\begin{array}{l}\text { Front. Observi la simetria facial en repòs. Demaneu al pacient que arrugui el } \\
\text { front i miri cap al sostre. }\end{array}$ & $\begin{array}{l}\square \text { Simetria Facial } \\
\square \text { Simetria de les arrugues }\end{array}$ \\
\hline & Ulls. Demaneu al pacient que tanque els ulls amb tota la força com pugui. & $\square$ Simetria de tancament parpelles \\
\hline & $\begin{array}{l}\text { Boca. } \\
\text { Demaneu que somrigui, i que li mostre els dents. } \\
\text { Demaneu que faci un mohín. }\end{array}$ & $\begin{array}{l}\square \text { Simetria llavis } \\
\square \text { Simetria arrugues del coll }\end{array}$ \\
\hline $\begin{array}{c}\text { PPCC IX } \\
\text { GLOSSOFARINGI }\end{array}$ & $\begin{array}{l}\text { "Reflex faringi (no més s'aplica quan no hi ha una correcta elevació del } \\
\text { paladar) } \\
\text { Demaneu al pacient que obri la boca i, amb l'ajuda d'un depressor lingual, } \\
\text { estimuli cada costat de la paret posterior de la faringe. }\end{array}$ & $\begin{array}{l}\text { 口 Contracció faringe, desplaçament } \\
\text { posterior de la llengua i sensació } \\
\text { nàusea }\end{array}$ \\
\hline \multirow[b]{2}{*}{$\begin{array}{l}\text { PPCC X } \\
\text { VAGUE }\end{array}$} & $\begin{array}{l}\text { Elevació del paladar tou } \\
\text { Demani al pacient que obri la boca i digui "a", el que produirà l'elevació del } \\
\text { vel del paladar. } \\
\text { Observi l'úvula (si la llengua no l'hi permet, abaixa-la amb l'ajuda d'un } \\
\text { depressor) }\end{array}$ & $\begin{array}{l}\square \text { Úvula en posició medial (simetria). } \\
\square \text { Elevació del paladar* }\end{array}$ \\
\hline & $\begin{array}{l}\text { Avaluació funció laríngia } \\
\text { Demaneu a pacient que hi faci una fonació llarga de la vocal /a/ } \\
\text { Demaneu al pacient que incrementi/disminuīa el de la vocal /a/ } \\
\text { Demaneu al pacient que hi faci un grunyit breu i agut. Demaneu que tussi. } \\
\text { Demaneu al pacient que conte fins } 300 .\end{array}$ & $\begin{array}{l}\square \text { Més de7-8 segons } \\
\square \text { Capacitat de modificar el to } \\
\square \text { Tancament glòtic: so és intens i } \\
\text { agut. } \\
\square \text { Esforç del mecanisme vocal }\end{array}$ \\
\hline \multirow{4}{*}{$\begin{array}{l}\text { PPCC XI } \\
\text { ESPINAL }\end{array}$} & Avalua la simetria dels músculs esternocleidomastoideos. & $\square$ Simetria \\
\hline & $\begin{array}{l}\text { Demaneu al pacient que giri el cap un costat i la mantingui en aquesta } \\
\text { posició. Mentrestant intenteu tornar el cap a la línia mitja. }\end{array}$ & $\square$ Debilitat lateral \\
\hline & Repetiu l'operació però el cap cap endavant. & $\square$ Debilitat anterior/posterior \\
\hline & Demaneu al pacient que alci les espatlles a contra resistència. & $\square$ Debilitat anterior/posterior \\
\hline \multirow{6}{*}{$\begin{array}{l}\text { PPCC XII } \\
\text { HIPOGLós }\end{array}$} & Avalua la llengua en repòs & $\begin{array}{l}\square \text { Atrofia } \\
\square \text { Fasciculacions }\end{array}$ \\
\hline & Demaneu al pacient que traga la llengua & $\square$ Punta llengua en línia mitja \\
\hline & Demaneu al pacient que mogui la llengua d'una comissura a l'altra. & $\square$ Velocitat adequada \\
\hline & $\begin{array}{l}\text { Demaneu al pacient que pressioni la llengua contra cadascuna de les galtes, } \\
\text { oposant-se l'explorador col-locant externament els seus dits. }\end{array}$ & $\square$ Debilitat cos de la llengua \\
\hline & Demaneu que pronunciī els fonemes linguals $/ r / / / \mathrm{i} / \mathrm{t} /$. & $\begin{array}{l}\square \text { Debilitat en l'elevació de la punta } \\
\text { de la llengua }\end{array}$ \\
\hline & Demaneu que pronuncii els fonemes linguals / k/ i/g/. & $\begin{array}{l}\square \text { Debilitat en l'elevació part } \\
\text { posterior de la llengua }\end{array}$ \\
\hline
\end{tabular}

Fig 3. Ficha diseñada para el estudio de los pares craneales involucrados en el habla.

(cc) EY-NC-ND 2018, Universitat Politècnica de València 


\section{Resultados}

El objetivo general del presente proyecto es realizar un análisis de la implantación de un proyecto de ApS en alumnos de segundo de Logopedia en comparación con alumnado que no recibió esta metodología docente. Tras la implantación del proyecto, el análisis de los datos obtenidos a partir de las fichas mostró que, de forma global, los estudiantes que habían participado en el proyecto ApS obtuvieron calificaciones significativamente superiores en la evaluación de la propedéutica clínica respecto al alumnado que no participó (Figura 3). El análisis de los ítems por separado (Figura 4) mostró que las competencias que mejoraron significativamente en los alumnos que implementaron el ApS eran las relacionadas con el secreto profesional, el trato del paciente, la comunicación oral con el paciente, y la empatía. Sin embargo, no se observaron diferencias significativas en cuestiones teórico-prácticas, como es la realización de una correcta exploración neurológica, que fue similar en ambos grupos (Figura 4).

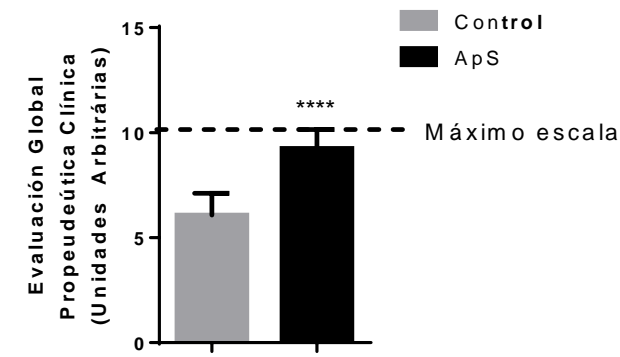

Fig 3. Comparación de las calificaciones obtenidas en alumnos que participaron o no en el proyecto ApS.

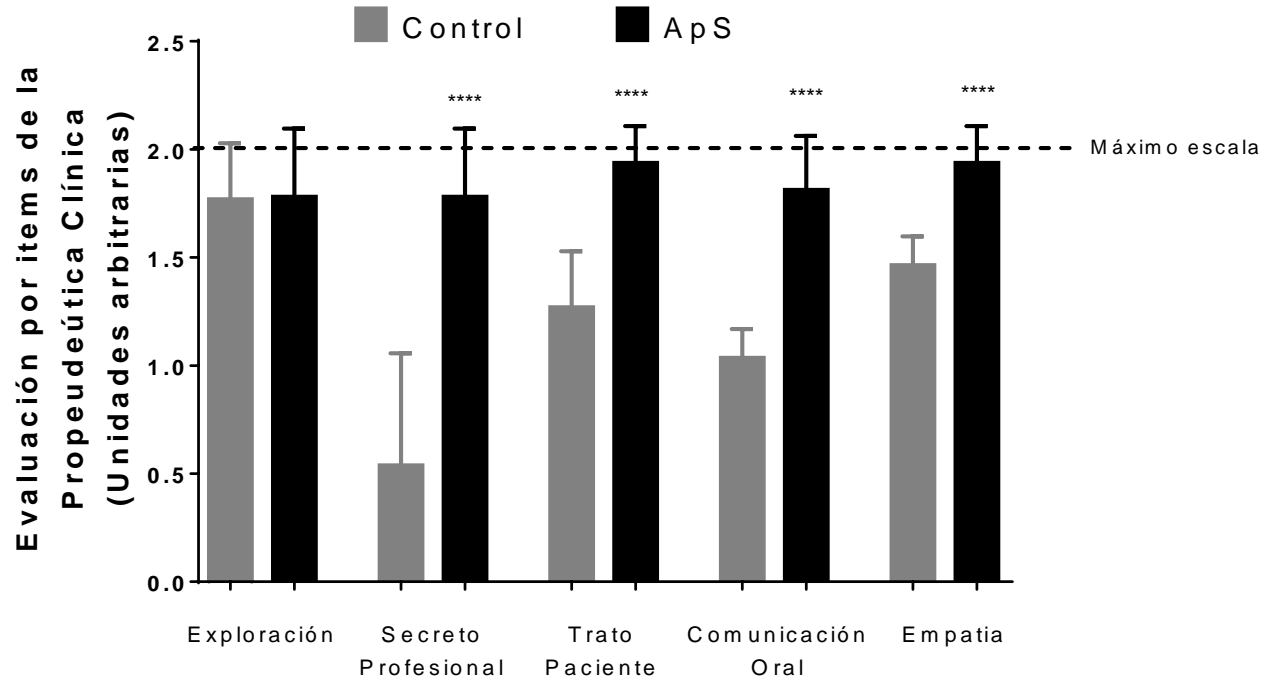

Fig 4. Comparación de las calificaciones obtenidas en los diferentes ítems en alumnos que participaron o no en el proyecto ApS. Los ítems utilizados fueron: exploración (explora de manera eficiente los pares craneales (PC) que participan en la inervación de la musculatura encargada del habla), secreto profesional (evita comentar información reservada que no sea estrictamente necesaria para el caso. Mantiene el máximo secreto profesional), trato paciente (trabaja de manera adecuada, cuidando de manera específica las formas y adaptándose al nivel y las características del paciente), comunicación oral (comunica de forma oral correctamente y fundamentada profesionalmente sus observaciones y conclusiones) y empatía (sabe escuchar sin interrumpir. Sabe interpretar el lenguaje no oral del paciente. Muestra autocontrol emocional en las interacciones. Observa y escucha activamente). 
Aplicación de la metodología ApS en alumnos del Grado de Logopedia: intervención con personas de la tercera edad.

En relación a la evaluación docente del proyecto, los alumnos colaboradores realizaron una encuesta acerca de su experiencia. Los resultados mostraron, como se puede ver en la Figura 5 , que los alumnos colaboradores no necesitaron cambiar las estrategias didácticas durante las diferentes intervenciones, y las actividades desarrolladas se diseñaron teniendo en cuenta las características y motivaciones de los alumnos de segundo.

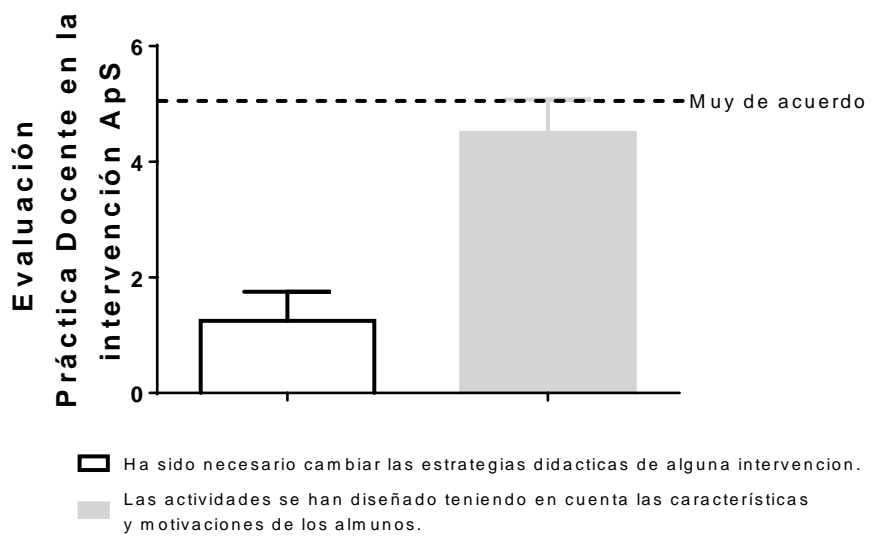

Fig 5. Evaluación de la práctica docente desarrollada durante la implantación del proyecto ApS. Encuestas realizadas por los alumnos colaboradores que participaron activamente en la implementación del proyecto.

Al finalizar la intervención, tanto los alumnos como las personas de la tercera edad realizaron encuestas de satisfacción (Figuras 6, 7 y 8). En cuanto a las personas mayores (Figura 6), las encuestas reflejaron que de forma general la experiencia había sido de su agrado, se sintieron cómodos con los alumnos y volverían a repetir.

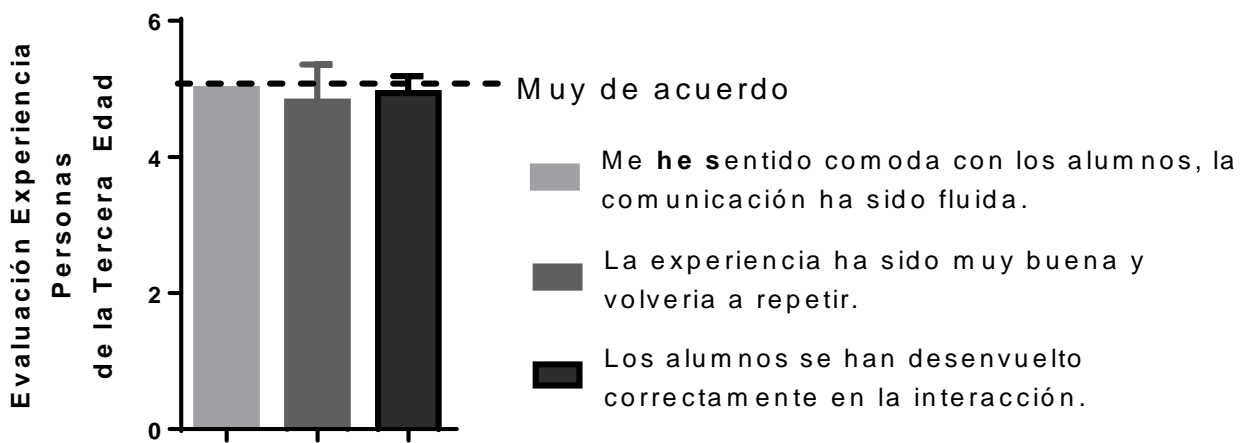

Fig 6. Resumen de los datos obtenidos en la encuesta de satisfacción realizada por las personas de la tercera edad subsidiarias del proyecto ApS.

En relación al alumnado que participó en la experiencia, la encuesta de satisfacción se dividió en dos categorías: evaluación de los elementos del curriculum y adecuación de las intervenciones planteadas durante la implantación del proyecto ApS. En relación a los

(cc) EY-NC-ND 2018, Universitat Politècnica de València

Congreso IN-RED (2018) 
elementos del curriculum desarrollados en el proyecto ApS, los estudiantes consideraron que en general habían mejorado sus competencias en la exploración neurológica (tanto en el MMSE (Minimental Test) como en la exploración de los PC involucrados en el habla), que el tiempo de desarrollo de las actividades había estado ajustado y que la estrategia didáctica basada en ApS les había fomentado el interés por aprender y hacer la propedéutica clínica de una forma correcta (Figura 7).

En relación a la adecuación de las intervenciones planteadas, se puede ver en la Figura 8 que los estudiantes consideraban que tenían las competencias necesarias para el desarrollo de las actividades, y que las intervenciones realizadas se ajustaban al curriculum de la asignatura. Además, valoraron de forma muy positiva la experiencia.

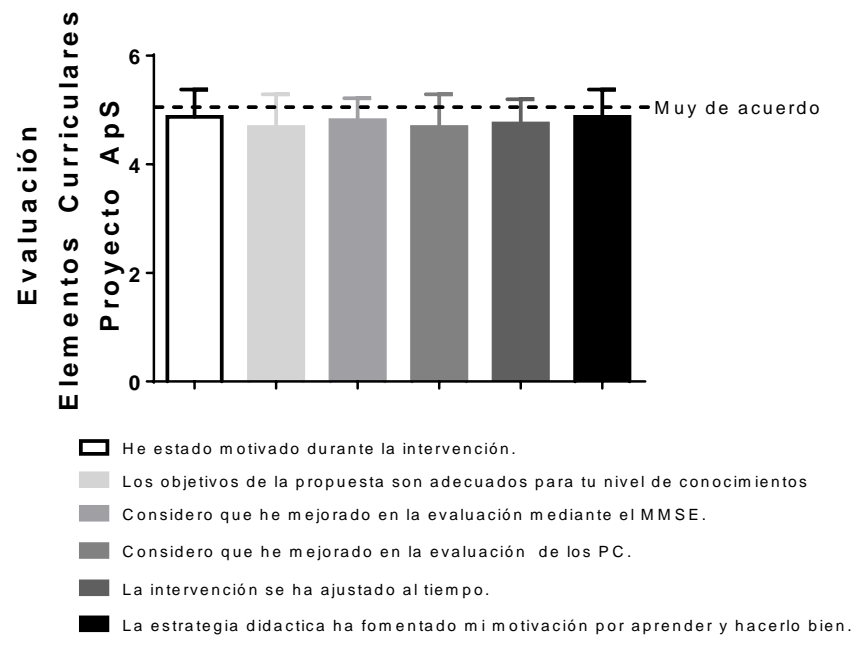

Fig 7. Resumen de los datos obtenidos en la encuesta que evaluaba los elementos del curriculum desarrollados en el proyecto ApS.

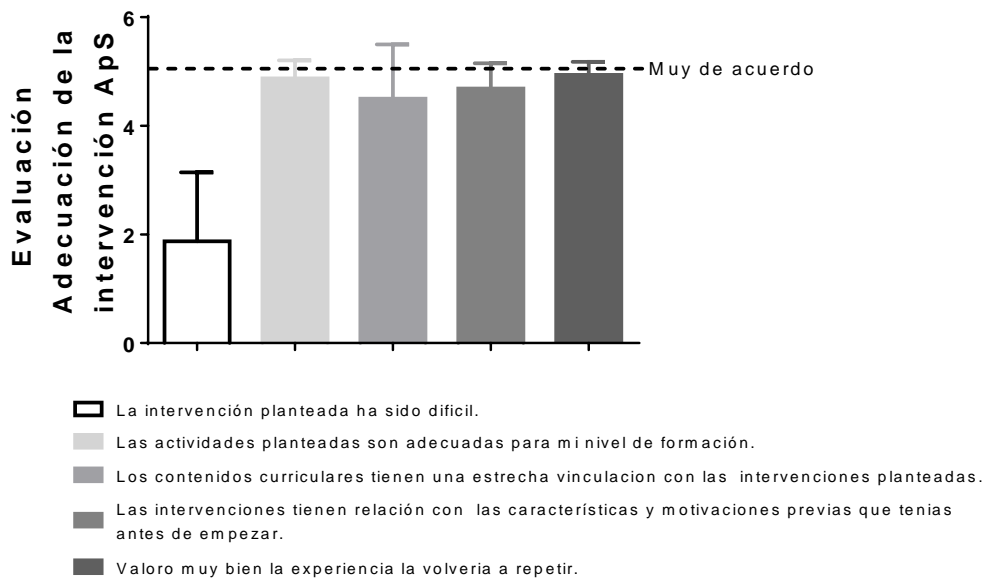

Fig 8. Resumen de los datos obtenidos en la encuesta que evaluaba la adecuación de la intervención de la intervención y de los contenidos curriculares del proyecto ApS. 


\section{Conclusiones}

La implantación del proyecto de ApS en los alumnos de segundo del Grado de Logopedia ha permitido abrir vías de encuentro entre la educación formal y la no formal en el Grado de Logopedia. Con el proyecto ApS se ha potenciado que el alumnado asuma las respuestas a las necesidades del entorno como responsabilidad compartida permitiendo que la UV se abra a la ciudad de Valencia. De forma específica, los alumnos de segundo de Logopedia que participaron en el proyecto ApS han reforzado sus competencias relacionadas con la evaluación y diagnósticos de las alteraciones del lenguaje en el envejecimiento y/o trastornos degenerativos. Además, la aplicación de la metodología de ApS en los alumnos potenció de manera significativa sus habilidades comunicativas, de secreto profesional y de empatía tan necesarias para el desarrollo de su profesión.

Cabe señalar que las personas de la tercera edad fueron evaluadas satisfactoriamente por parte de los estudiantes, se les realizó un diagnóstico de sus posibles alteraciones relacionadas con el habla y se les aconsejó con el fin de mejorar su calidad de vida. Todo ello trajo de la mano que tanto estudiantes como jubilados fueran participes de un intercambio de conocimientos intergeneracional, favoreciendo así competencias relacionadas con la comunicación oral y empatía en los estudiantes.

Por otro lado, los estudiantes colaboradores y gracias al proyecto ApS, se iniciaron en competencias relacionadas con el desarrollo de liderazgo, toma de decisiones y nuevas metodologías de enseñanza-aprendizaje.

\section{Referencias}

CASADO DE LA GALA, L. (2015). Aprendizaje servicio, proceso de mejora para la ciudadanía. Palencia: Facultad de Educación de Palencia, Universidad de Valladolid.

MARTÍNEZ, M. et al. (2010). Aprendizaje servicio y responsabilidad social de las Universidades. Barcelona: Ediciones Octaedro, S.L. y Universitat de Barcelona.

PUIG, JM., BATLLE, R., BOSCH, C., PALOS, J. (2007). Aprendizaje servicio: educar para la ciudadanía. Madrid: Ministerio de Educación y Ciencia. Centro de Investigación y Documentación Educativa. Ediciones Octaedro.

\section{Legislación y normas}

España. Orden CIN/726/2009, de 18 de marzo, por la que se establecen los requisitos para la verificación de los títulos universitarios oficiales que habiliten para el ejercicio de la profesión de Logopeda. BOE. 26 de marzo de 2009, núm. 73, p. 29159-29163.

España. Real Decreto 592/2014, de 11 de julio, por el que se regulan las prácticas académicas externas de los estudiantes universitarios. BOE. 30 de julio de 2014, núm. 184, p 8138.

Universitat de Valencia. Resolució de 3 d'abril de 2017, del Vicerectorat de Polítiques de Formació i Qualitat Educativa, per la qual es convoquen les ajudes per al desenvolupament dels projectes d'innovació educativa i millora de la qualitat docent per al curs 2017/2018.

(cc) EY-NC-ND 2018, Universitat Politècnica de València

Congreso IN-RED (2018) 